\title{
Excellent Crystal Coloration and An Extraordinary Improvements of Developing Synthetic Quartz Single Crystals Growth and Defects
}

\author{
Loai Aljerf ${ }^{1 *}$ and Nuha AlMasri ${ }^{2}$ \\ ${ }^{1}$ Department of Basic Sciences, Faculty of Dental Medicine, Damascus University, Damascus, Syria \\ ${ }^{2}$ Department of Chemistry, Faculty of Medicine, Syrian Private University, Damascus, Syria
}

Received: 㭗July 21, 2018; Published: 紫July 27, 2018

*Corresponding author: Loai Aljerf, Department of Basic Sciences, Faculty of Dental Medicine, Damascus University, Damascus, Syria, Tel: +96394448203; Email: envirochrom@hotmail.com

\begin{abstract}
The analysis of the impurity content crystals grown in sodium carbonate solution is carried out using flame technique. Colored crystals have been produced from aqueous solutions of potassium carbonate under laboratory conditions and using a steel autoclave. The seeds were slices cut parallel to the planes (0001). With the impurity of aluminum and irradiation, defective color centers have generated quartz coloration phenomenon. This is occurred on the base of the electrical balance by exchanging aluminum ions with tetravalent silicon ions in the presence of alkali elements (monvalent) i.e. $\mathrm{Na}^{+}$or $\mathrm{Li}^{+}$. Interestingly, the current paper introduces a method suggests the utilization of silica-rich rocks to develop the growth of large crystals of synthetic quartz.
\end{abstract}

Keywords: Metallic impurity; Quartz; Crystalline; Piezoelectric; Aluminum; Flame photometric emission

\section{Introduction}

Until recently, all the quartz required for the production of oscillator crystals for frequency control has been obtained from natural resources. Although quartz is one of the most abundant minerals in the earth's crust, it is only found in large crystals of the required quality in a few isolated regions. During the past 20 years, work has been carried out to develop processes for the controlled growth of quartz in the laboratory $[1,2]$. Considerable success has been obtained by many teams [3], crystals of piezoelectric-quality weighing over $1 \mathrm{lb}$. having been grown [4]. All the modern processes used for the growth of synthetic quartz have been developed by pioneering works $[5,6]$ in the current century. Although the methods adopted by various workers in this field are basically similar, there are certain fundamental differences which affect the properties of the crystals. Because of its glass forming properties and its allotropic modifications it is not possible to grow quartz from the vapor or the melt. Growth from solution must be used and as quartz is virtually insoluble in aqueous media under ordinary ambient conditions it is necessary to use elevated temperatures and pressures to obtain sufficient solubility. These so-called hydrothermal conditions are probably similar to those in which much natural quartz has been formed. At temperatures approaching $400^{\circ} \mathrm{C}$ and pressures of 1000 atm (about 7 tons/in. ${ }^{2}$ ) quartz is readily soluble in alkaline solutions such as sodium carbonate.

\section{Materials and Methods}

\section{Crystal Growing Technique}

A schematic diagram of the apparatus used by the authors is shown in Figure 1. In what will be called the standard process, a steel autoclave constructed to withstand high pressures has seed crystals suspended from the lid and a supply of crushed meltinggrade quartz at the bottom. The autoclave is about $80 \%$ filled with a solution containing $88 \mathrm{~g} / \mathrm{L}$ of sodium carbonate and sealed. The simple furnace used consists of a hotplate on which the autoclave stands surrounded by micaceous-flake thermal insulation. By this means a temperature gradient is established so that it is hotter at the bottom in the region of the nutrient crushed quartz than at the top where the seeds are located. Under the working conditions, the autoclave is filled with a single-phase fluid. The pressure developed being a function of the temperature and of the percentage of the space originally occupied by the solution at room temperature. The 
temperature at the base of the autoclave is controlled at about $400^{\circ} \mathrm{C}$ and the temperature at the seeds reaches equilibrium some $40^{\circ} \mathrm{C}$ lower. The temperature gradient along the length of the autoclave is not uniform, a fall of about $20^{\circ} \mathrm{C}$ occurring across the metal at the base and most of the remaining drop being across the nutrient. The space above the nutrient is approximately isothermal and the supersaturation in this region remains constant. Thus, crystals can be grown at approximately the same rate of growth in any part of the autoclave.

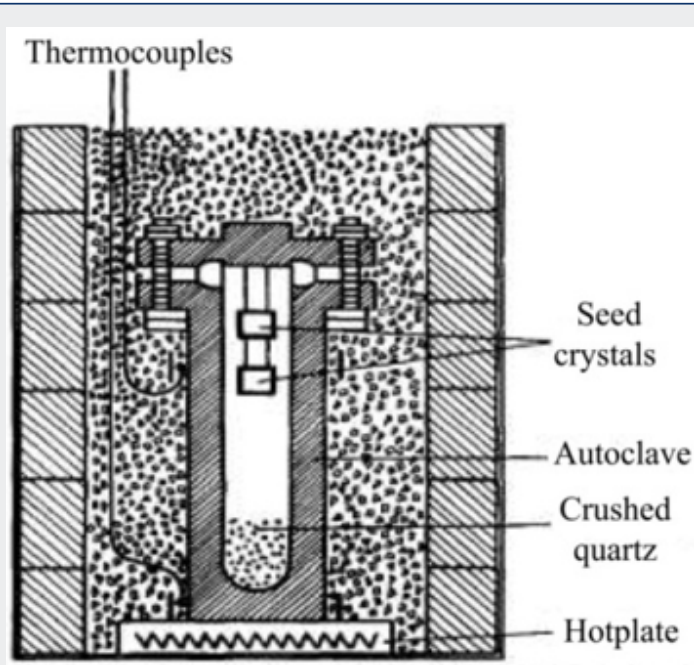

Figure 1: Diagram of apparatus for the growth of synthetic quartz.

For a given design of the autoclave, the rate of growth is dependent on seed orientation, pressure, temperature and temperature differential. The seed orientation which has been adopted in most of the work to be described is the basal plane or Z-cut. Figure 2 shows the relation between this cut and the minor rhombohedral or T-cut which has also been used as a seed for the growth of synthetic quartz. It is shown subsequently that the seed orientation not only affects the rate of growth but also has a marked effect on the way in which impurities are incorporated in the crystal. It is most convenient to control the rate of growth by means of the temperature difference and this is done by adjusting the flow of solution but not exceed a certain maximum, which, for the conditions used in the standard process, is about $0.5 \mathrm{~mm} /$ day on each side of the seed measured in the direction of the optic axis. In fact, the visual quality is in some respects a misleading criterion and the measurements of the mechanical damping recorded in Table 1 ; show that a progressive improvement in crystalline perfection takes place as the growth rate is reduced. As it happens, for most practical applications the quality corresponding to $0.5 \mathrm{~mm} /$ day is adequate but for especially stringent requirements it may be necessary to employ a lower growth rate or an alternative growing technique. A small pilot plant has been set up to grow crystals by the standard process. A growth of $15 \mathrm{~mm}$ takes place in a period of about a month, the resulting crystals weighing about $135 \mathrm{~g}$.

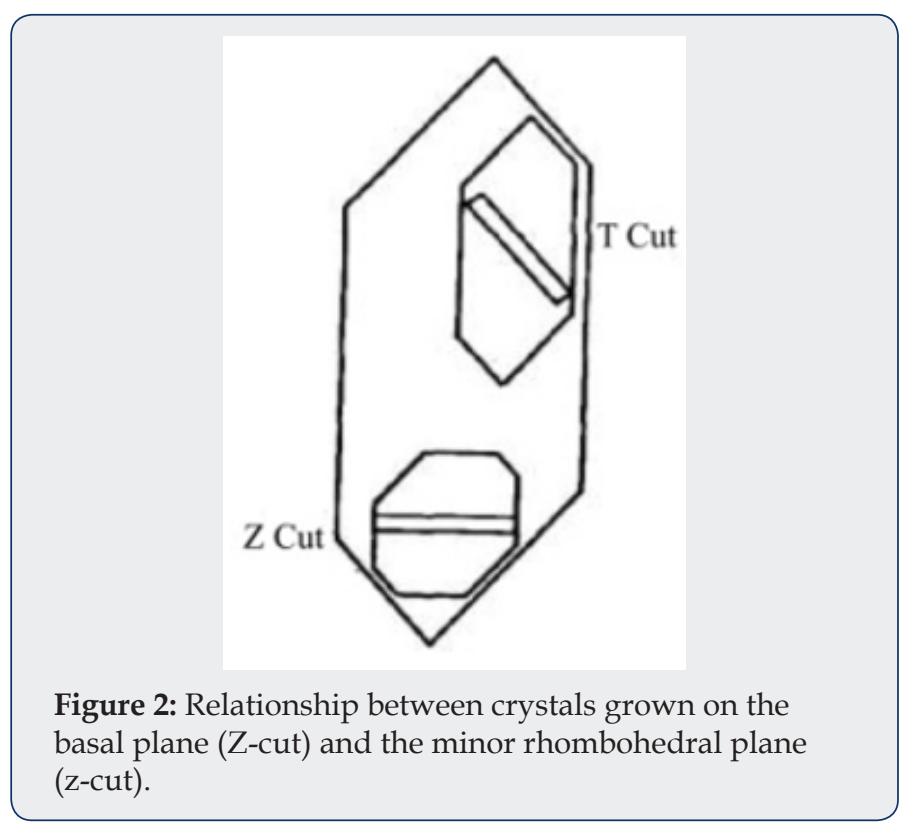

Table 1: Mechanical damping of quartz

\begin{tabular}{|c|c|c|}
\hline \multirow{2}{*}{ Material } & $\begin{array}{c}\text { Growth rate (mm/ } \\
\text { day) }\end{array}$ & Damping ratio* \\
\hline \multirow{3}{*}{ Natural Synthetic } & 0.55 & 30 \\
\cline { 2 - 3 } & 0.38 & 14 \\
\cline { 2 - 3 } & 0.25 & 5 \\
\hline
\end{tabular}

* The damping measured [7] at $20^{\circ} \mathrm{C}$ using a special design of oscillator crystal.

\section{Results and Discussion}

\section{Influence of impurities}

The standard process recrystallizes a low-grade quartz-which due to size and imperfections such as twinning is unsuitable for piezoelectric use into crystals of a size and quality which are ideally suited to this purpose. However, the melting grade quartz used as nutrient still has to be imported and considerable work has been carried out over the last 5 years to develop processes which can be used with relatively impure nutrient materials. Reasonable success has been obtained using flint and a variety of quartzites. Early in this investigation it was found that quartz could be grown on Z-cut seeds using impure nutrient materials, but that the quality, particularly of large crystals, was not good enough for piezoelectric use. It is now known that the poor quality is due to the incorporation of impurities in the synthetic crystal during growth. However, it was found that it was possible to grow crystals of piezoelectric quality by modifying the solution in which the crystals were grown. Good crystals have been grown from flint and impure quartzite by using a solution containing $40 \mathrm{~g} / \mathrm{L} \mathrm{Na}_{2} \mathrm{CO}_{3}, 33 \mathrm{~g} / \mathrm{L} \mathrm{NaOH}$, and 3.4g/L NaF. Further examination of a number of different quartzites showed that many of them could be used satisfactorily with the standard process; these are referred to as class A quartzites. It is now known that the difference between class A and class B quartzites (class B 
being those quartzites which require the modified process) lies in the type of accessory minerals which are associated with the quartz. In particular, the structure of the feldspar, which is commonly the primary accessory mineral in quartzites, plays an important role in deciding whether the material will be class A or $\mathrm{B}[7,8]$.

Work by Dickson et al. [9] using a paramagnetic resonance technique has shown that aluminum is the impurity which affects the quality of synthetic quartz crystals grown from class B materials using the standard sodium carbonate solution. This result has been corroborated by spectrographic analysis on a number of specimens. In addition, the direct test has been made by deliberately adding aluminum in a number of forms along with a pure quartz nutrient. Such adulterated quartz now acts as a class B nutrient and crystals grown from it using the standard solution have the habit and poor quality of a crystal grown under similar conditions from flint or a class B quartzite. Further, by using the modified solution, the defects can again be overcome. Figure 3 shows four crystals which illustrate this result. The crystals from left to right were grown from nutrients and in solutions. The most notable feature which can be seen from the photograph is the nature of the growth surface approximating to (0001). This surface is smooth on the crystals grown from quartz in sodium carbonate solution and from quartz with aluminum in the modified solution, but it is rough and pitted in the case of the crystals grown from flint and from quartz with aluminum, in sodium carbonate solution. It will be shown that the nature of the growth surface is closely related to the manner in which aluminum is incorporated in synthetic quartz grown on Z-cut seeds.

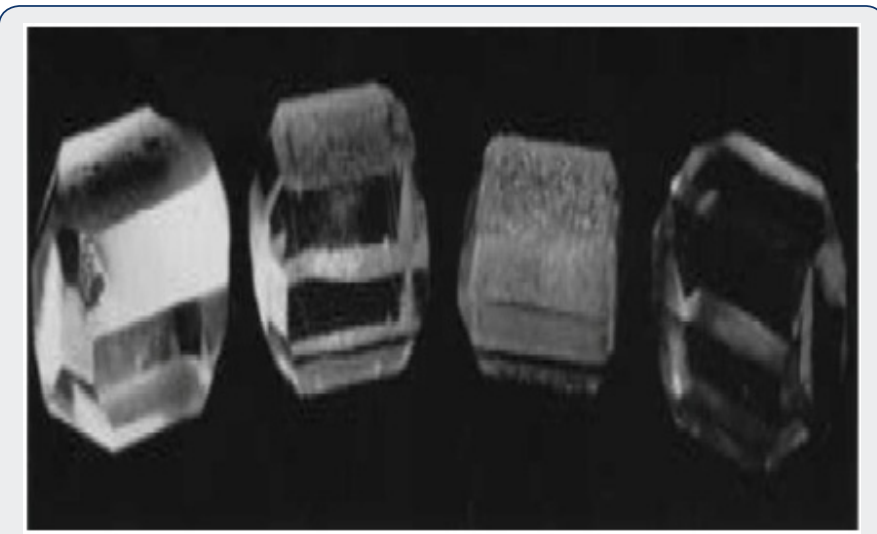

Figure 3: Crystals grown to illustrate the importance of aluminum as an impurity in low-quality nutrients.

It has been known for some time that when natural quartz is irradiated with X-ray or any other ionizing radiation, the material darkens. Grasse et al. [10] studied this phenomenon in detail and showed that the darkening produced in natural quartz is often non-uniform showing a banded structure. The darkening occurs in sheets parallel to the major rhombohedral planes and is therefore connected with the growth of the crystal, probably being associated with changes in the environment in which the crystal grew. When large crystals of synthetic quartz were first grown by the authors, their behavior under X-irradiation was determined. Figure 4 shows the result of irradiating an X-cut section of a synthetic quartz crystal grown on a Z-cut seed. It can be seen that the central region corresponding to the natural quartz seed has darkened uniformly and that there are two regions beneath the minor rhombohedral faces which have also darkened rather more intensely than has the seed. The remainder of the synthetic growth has not darkened under this dose. The diagram in Figure 5 shows the region under the minor rhombohedral face on a larger scale. The triangular region abc corresponds to growth which has taken place on the minor rhombohedral face as it develops. Spectrographic analyses of material taken from various regions of a number of crystals grown by the standard process have shown that the total aluminum concentration in the growth on the Z-cut orientation is commonly less than 40 parts in $10^{6}$ atomic replacements. However, in the growth under the minor rhombohedral face the aluminum content may be 10 times this figure, i.e. 400 parts in $10^{6}$. The impurity content of the melting-grade nutrient lies between these two figures and the low aluminum concentration in quartz grown on a Z-cut seed is in part due to the "scavenger action" of the growth on the minor rhombohedral faces which are formed during the growth. This also illustrates a general result found in the growth of synthetic quartz, namely that it is easier to introduce impurities during growth on the minor rhombohedral face than on the basal plane.

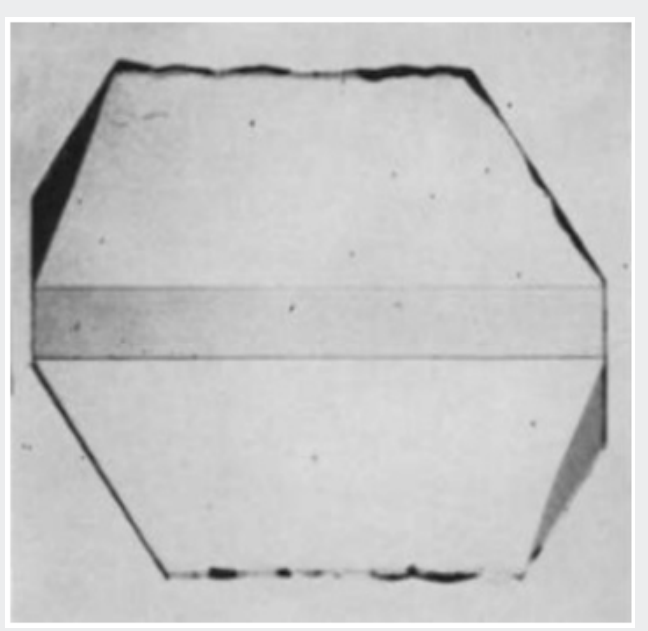

Figure 4: Photomicrograph of an X-cut section from a synthetic quartz crystal grown on a Z-cut seed from a pure melting-grade quartz nutrient after $\mathrm{X}$-irradiation.

If, however, there is a large excess of aluminum in the system, it is found that the crystals grown on the basal plane will darken readily in a characteristic way as shown in Figure 6. In addition to the darkening of the seed crystal and the growth under the minor rhombohedral face, there is darkening of the primary growth in the form of distorted narrow-angled cones directed along the 
c-axis. These cones terminate in the rough, pitted growth surface and are apparently associated with the pits. This can also be seen from the bands which run parallel to the seed surface. These bands which are regions of either more or less intense darkening than the surrounding material are reproduced precisely on both sides of the seed. It can, therefore, be deduced that these bands are produced either by changes of the temperature or pressure in the autoclave or, what is more likely, by changes in the concentration of aluminum in the solution. These bands will therefore represent the nature of the growth surface at the particular time when they are formed. The discontinuities in these bands tend to follow the boundaries between the cones of darkening.

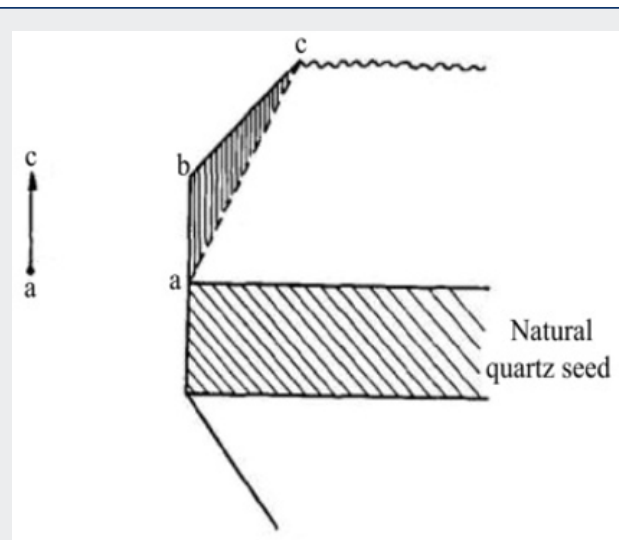

Figure 5: Diagram showing the $X$-ray darkening of quartz deposited in the accessory growth on the minor rhombohedral face.

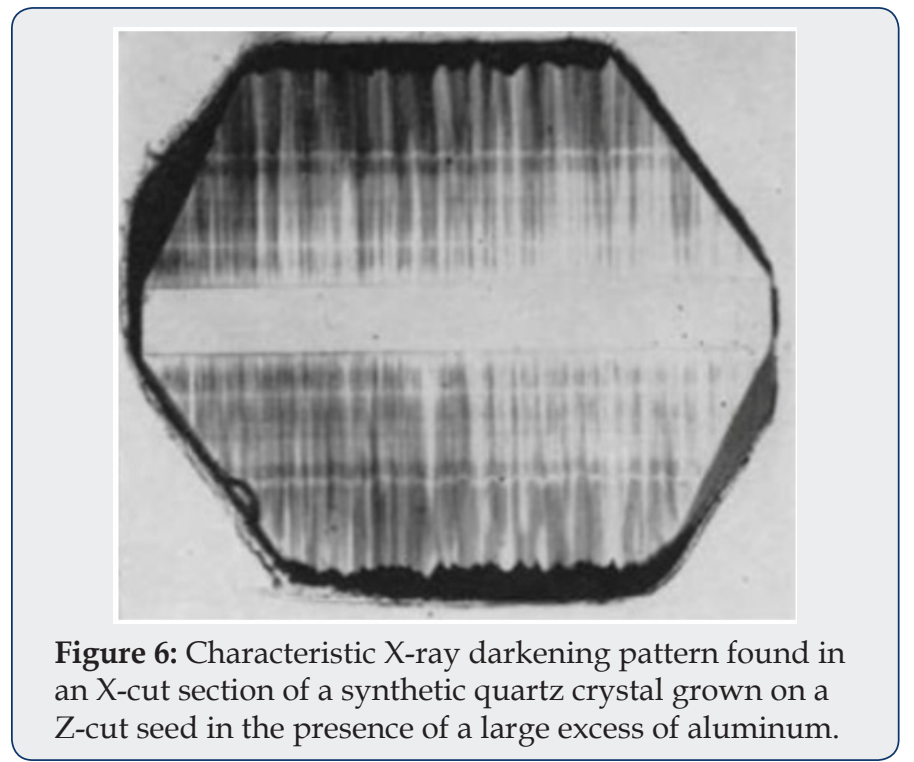

\section{Mechanism of growth}

The characteristic cone darkening structure can be used to obtain a model for the mechanism of growth on the basal plane. This is illustrated in Figure 7. It is assumed that growth takes place independently on a large number of centers in contrast to growth on a habit face where only a limited number of centers are active and growth takes place by sheets spreading across the growth surface. The growth centers on the basal plane may be associated with spiral dislocations but there is, as yet, no direct evidence for growth spirals. If it is now assumed that quartz is deposited on the individual centers at different rates it will be seen that those which are growing faster overtake their more slowly growing neighbors and render them inactive. In the schematic diagram in Figure 7, all nine centers are active at the beginning of the growth; at later stage only centers 2, 4, 6 and 8 are active and eventually only centers 4 and 6. If the aluminum incorporation is uniform on any one growth centre but differs from one to the next, it will be seen that this gives rise to the characteristic darkening pattern. Figure 7 also shows photomicrographs of the growth surfaces at various stages in the growth together with darkening patterns at similar stages for sections cut perpendicular to the c-axis. The individual "cobbles", which are the termination of the growth cones in the surface of the crystal, and the tine structure in the darkening patterns both tend to become coarser as the growth proceeds. This is in agreement with the suggested growth mechanism.

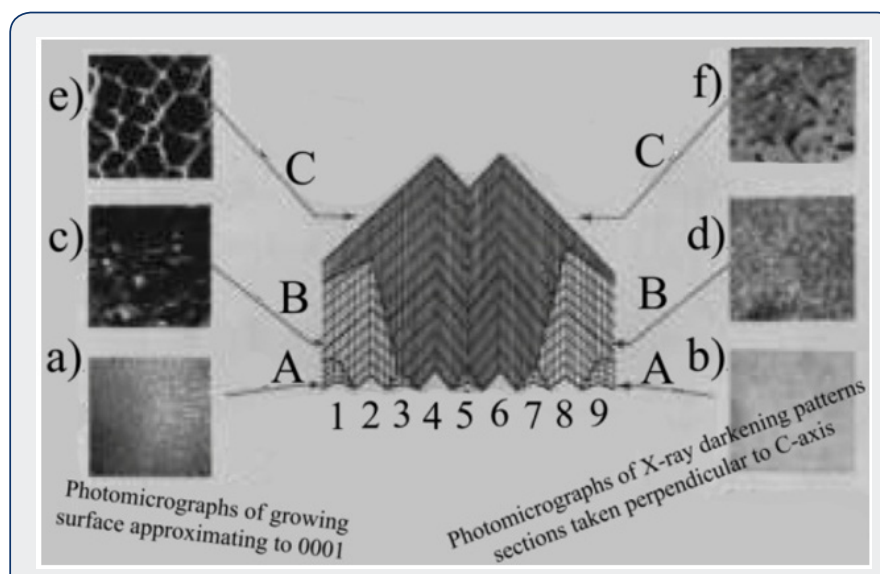

Figure 7: Illustrating the mechanism of growth of synthetic quartz on Z-cut seeds.

The nature of the rough, pitted growth surface obtained when quartz is grown on the basal plane in the presence of a large excess of aluminum cannot be directly explained in terms of the suggested mechanism. Figure 8a is a photomicrograph of the surface and shows clearly that the pits have no obvious crystallographic orientation. However, crystals have been grown with only just sufficient aluminum present in the system to commence the incorporation of aluminum by the above mechanism. In this case, the aluminum apparently only goes into the growth on isolated centers and produces a growth surface of the type shown in Figure $8 b$. It will be seen that certain of the larger cobbles have triangular pits at their centre. The relation between these pits and the rough growth surface produced by a large excess of aluminum is shown in Figure 8c and 8d. Figure 8c shows the growth surface of a crystal grown in the presence of a somewhat larger concentration of 
aluminum than that of Figure $8 \mathrm{~b}$. The pits are here more numerous and are commencing to overlap so that they interfere and lose their obvious crystallographic orientation. The crystal shown in Figure $8 \mathrm{~d}$ has a rough surface similar to that in Figure $8 \mathrm{a}$ and has been lapped to remove most of the disturbed surface. The bottoms of the pits of the rough surface show approximately the same form as those in Figure 8b. The characteristic rough surface is, therefore, formed from a large number of pits which overlap and interfere until the shape and symmetry of the pits is completely lost.

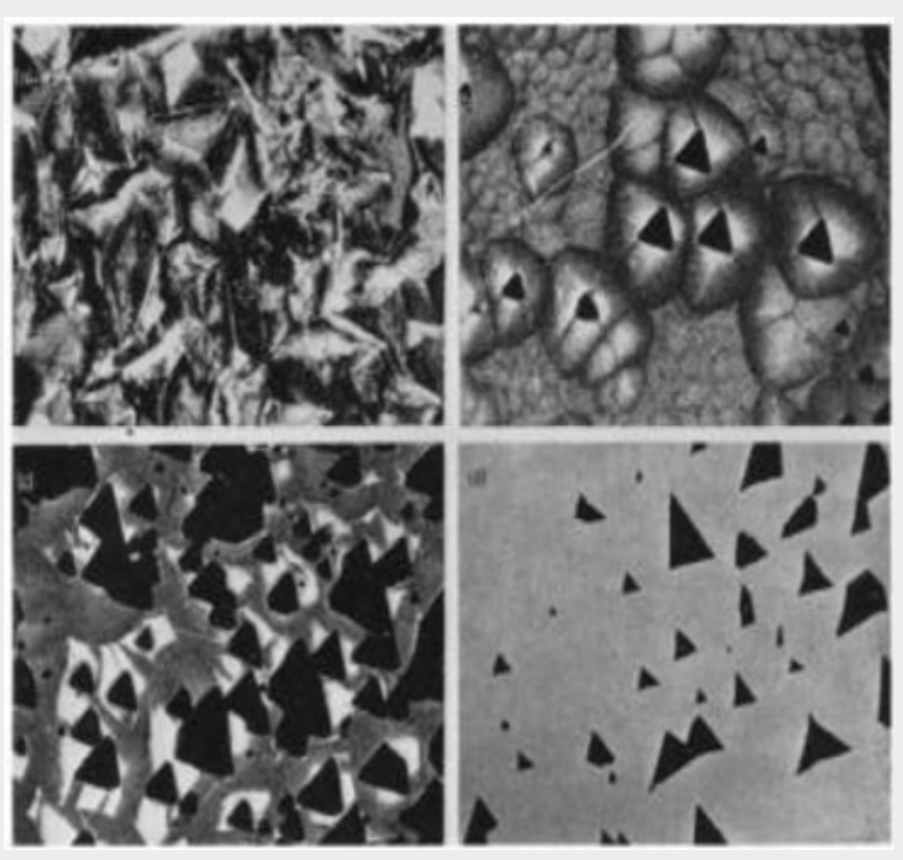

Figure 8: The nature of the rough growth surface formed in the presence of a large excess of aluminum. (a) Rough growth surface. (b) Isolated pits. c) Interfering pits. d) Rough surface after most of the damage has been removed by lapping.

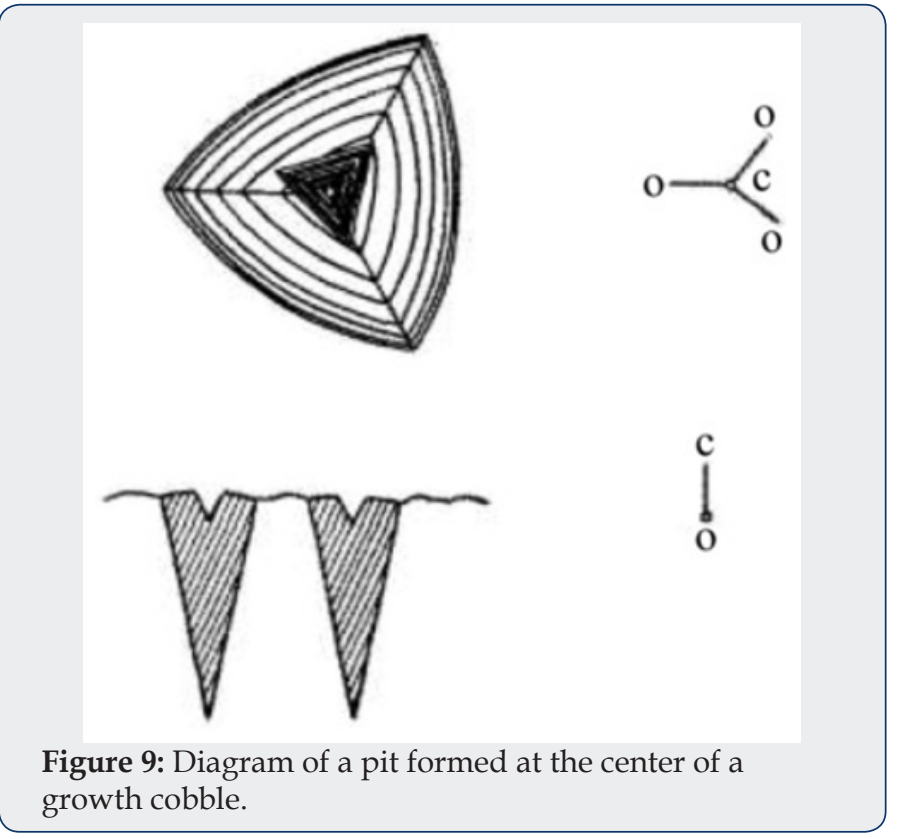

The nature of the individual pits is more readily seen by reference to Figure 9, which shows a diagram of a single cobble and its pit. The sides of the pit form reasonably flat faces which have been indexed using a microscope. The reason for the formation of such a high index face, if it is a true face, in the presence of excess aluminum is not understood. Figure 9 also shows a section containing the c-axis. If as appears likely, the pit is associated with the incorporation of aluminum, on irradiating such a section the growth cones giving rise to the cobbles with pits at their centers will darken readily. This is shown diagrammatically in Figure 9 while Figure 10 shows a photomicrograph which clearly illustrates this phenomenon. It has been seen that when crystals are grown in a large excess of aluminum, this aluminum is not incorporated uniformly in the crystal as it grows but is taken up preferentially on certain centers at the expense of the neighboring centers. As the lattice spacing will be a function of the aluminum concentration, it is reasonable to expect that strains will be set up at the boundaries between regions of different aluminum content. This strain can be seen readily by examining sections cut either parallel to or perpendicular to the c-axis in a polarizing microscope between crossed nicols. Parallel to the c-axis there are deep fissures found under the rough growth surface where the stress exceeds that necessary to produce fracture. Instead of the crystal appearing dark as it should in the extinction position, the field is crossed by bands of light and dark produced by the strain. The same phenomenon is, perhaps, more readily studied by examining sections cut perpendicular to the c-axis. Depending on the angle between the polarizer and analyzer the section should appear to be a uniform color when viewed in white light. Instead, a section cut from a 
crystal which has non-uniform aluminum incorporation will have a mottled appearance. This is shown in Figures 11 \& 12, which are photomicrographs of two sections of the same crystal, Figure 11 being taken near the seed crystal and Figure 12 near the end of the growth. This again illustrates the way in which the number of active growth centers decreases during the growth. In this particular example, the density of active centers has decreased by a factor of the order of 20 in about $5 \mathrm{~mm}$ of growth along the c-axis.

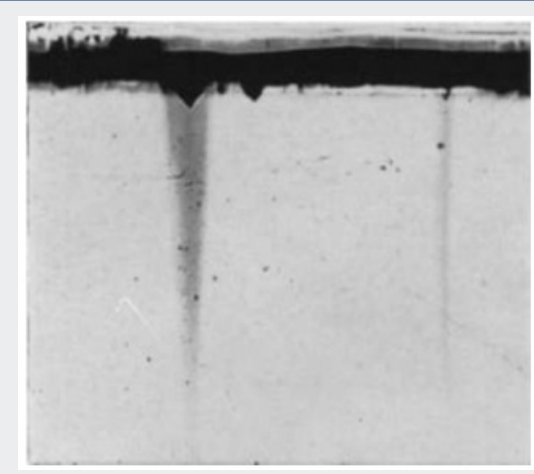

Figure 10: Photomicrograph of an X-cut section showing the relationship between the cones of darkening and the pits.

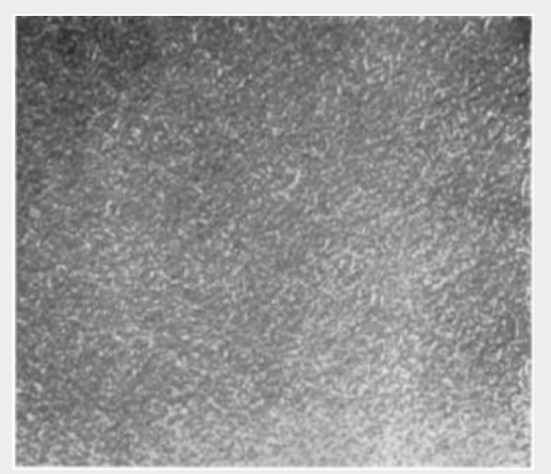

Figure 11: Strain pattern observed in crossed nicols in a Z-cut section of a crystal containing a large excess of aluminum (Section taken near seed/growth interface).

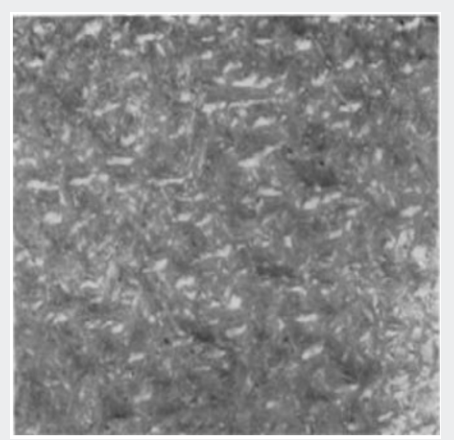

Figure 12: As Figure 11, Section taken near end of growth.

Comparison of the results of experiments in which crystals are grown on Z-cut seeds in the presence of high and low concentrations of aluminum shows that the mechanism by which aluminum is incorporated in the growing crystal is dependent on the concentration of impurity in the system. This has been studied more closely by a set of controlled experiments in which the concentration of aluminum, added as y-alumina, was steadily increased. Six crystals grown in the presence of aluminum deliberately added to the nutrient in proportions varying from 0.05 to $2.50 \%$ by weight are shown in Figure 13 . The concentration of aluminum added to the nutrient for the six crystals numbered 1 to 6 from left to right is given in the caption. Examining the nature of the growth surface, it will be seen that the first two crystals show no obvious signs of the inclusion of aluminum. By contrast, crystals 3-5 show the characteristic rough growth surface, crystal 5 being so strained that the growth is hardly single crystalline. With $2.5 \%$ aluminum added to the nutrient (crystal 6) all growth is prevented. Further experiments carried out using concentrations of aluminum in the range $0.10-0.25 \%$, show that the results are not consistent, in that a number of experiments carried out with the same aluminum concentration sometimes give a rough growth surface corresponding to non-uniform impurity incorporation and at other times give the smooth cobbled surface of the pure crystal. This behavior can be explained in terms of the suggested growth mechanism as follows in this paragraph. For low concentrations of aluminum in the system, the incorporation apparently takes place uniformly. Most of this aluminum is probably interstitial as the material only darkens slightly under X-irradiation. For high concentrations, it has been seen that the aluminum is taken up preferentially on certain growth centers. This can be understood, when it is considered that the energy required introducing an impurity atom is a function of the number of impurity atoms already incorporated in the growth on this centre. As the number of impurity atoms incorporated increases, the distortion of the lattice becomes greater and it becomes easier to include more impurity atoms. Thus, once the concentration of aluminum in the growth on a given centre exceeds a certain figure, further aluminum atoms will tend to be taken up preferentially on this centre at the expense of the neighboring centers. In a physical system of this type, the probability that non-uniform inclusion will take place and is a rapidly changing function of the concentration of impurity in the nutrient. It can be seen that this qualitative analysis explains the observations. For low concentrations of aluminum, the probability of non-uniform take-up is very small. As the concentration is increased the stage is reached where there is a reasonable chance that the aluminum is incorporated non-uniformly. This is the region where the results will not be consistent. At still higher concentrations, non-uniform inclusion will be the rule.

Specimens cut from the crystals shown in Figure 13 have been analyzed for aluminum and sodium using spectrographic and flame photometric techniques, respectively. The results are given in Table 2 in terms of the percentage atomic replacement of silicon by these elements have showed that the concentrations of sodium and aluminum are of the same order for low concentrations of aluminum added to the nutrient. This can be attributed to the sodium content tendency to saturate while the aluminum content 
continues to increase. It seems reasonable to conclude that the substitutionally added aluminum is associated with a sodium atom situated interstially. This centre would be responsible for the visible darkening produced by X-irradiation [11], a model consistent with that suggested by Ratheneau [12]. The excess aluminum found when the concentration added to the nutrient is large, could be present either as interstitial atoms or as two substitutional atoms associated with an oxygen vacancy. There is, at present, no evidence to distinguish between these two alternatives. In discussing the incorporation of impurities in synthetic quarts, some mention must also be made of work which has been carried out in attempts to include impurities other than aluminum [13]. It is well known that small monovalent ions in particular lithium and sodium can readily be introduced into the quartz lattice under the action of an electric field. These ions lie interstitially in the "tunnels" which are parallel to the c-axis in the quartz structure. It has already been shown that sodium is present in all synthetic quartz grown by the authors. Attempts have been made to introduce a number of other elements which might be expected to substitute for silicon in the lattice. In general, it has been found extremely difficult to introduce impurities into quarts grown on the basal plane. This result would appear to be different from that found by other workers in this field who have used seeds cut parallel to the minor rhombohedral face. In addition to aluminum, attempts have been made to incorporate the elements in growth on Z-cut seeds. Of these elements only, germanium has been successfully incorporated. Bearing in mind the similar ionic radii of silicon and germanium, it is not surprising to find that germanium will readily go into quartz as a substitutional impurity. Large amounts of germanium can be taken up by the quartz lattice without setting up measurable strain. As would be expected, the centre is not sensitive to X-irradiation. It is interesting to note that boron is not taken up, although its small size and its valence of three would, at first sight, make it an ideal atom for incorporation in the quartz lattice. No explanation is known for this behavior.

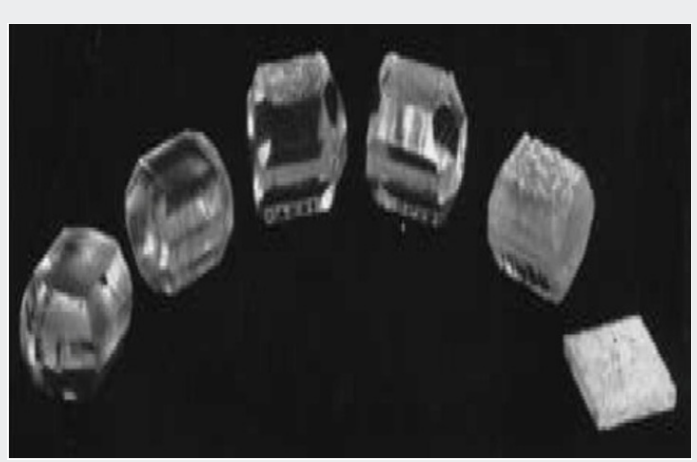

Figure 13: Crystals grown in the presence of increasing concentrations of aluminum. Aluminum by weight of quartz nutrient: (1) $0.05 \%$, (2) $0.125 \%$, (3) $0.25 \%$, (4) $0.50 \%$, (5) $1.25 \%$, and (6) $2.50 \%$.

\section{Coloration}

A striking example has been the growth of intensely colored emerald green quartz, a variety which does not occur naturally.
From the circumstances in which this crystal was grown, it has been deduced that the coloration is produced by the presence of a trace of chromium [13]. This diagnosis has still to be confirmed. The coloration in this crystal is very stable, being unaffected by heat treatment up to the $a-\beta$ inversion temperature or by prolonged $\mathrm{X}$-irradiation. Preliminary transmission measurements show that the material has an apparent cutoff in the ultraviolet at $2800 \AA$. It appears likely that this synthetic material is not related to the "greened" amethyst described by Samoylovich [14].

Table 2: Sodium and aluminum concentrations in synthetic quartz grown in $\mathrm{Na}_{2} \mathrm{CO}_{3}$ solution on $\mathrm{Z}$-cut seeds in the presence of aluminum.

\begin{tabular}{|c|c|c|}
\hline $\begin{array}{c}\text { Concentration of } \\
\text { aluminum added to } \\
\text { nutrient (weight per } \\
\text { cent) }\end{array}$ & \multicolumn{2}{|c|}{$\begin{array}{c}\mid 2 \\
\text { Imper cent) }\end{array}$} \\
\hline & Sodium & Aluminum \\
\hline- & 0.0050 & 0.0045 \\
\hline 0.05 & 0.0045 & 0.0065 \\
\hline 0.125 & 0.0170 & 0.0450 \\
\hline 0.25 & 0.0370 & 0.0650 \\
\hline 0.50 & 0.0390 & 0.1100 \\
\hline 1.25 & 0.0390 & 0.2900 \\
\hline 2.50 & - & - \\
\hline
\end{tabular}

\section{Conclusion}

The recent investigation, still incomplete, has shown that the nature of the cation in the solution from which the crystals are grown can have a considerable effect on the way in which impurities are incorporated in synthetic quartz. An investigation of the type described can be of value to the worker studying color centers in quartz in a number of ways.

a. The process described for the growth of large crystals of synthetic quartz can provide material with total impurity content, and particularly substitutional aluminum content, lower than is found in natural quartz. This is of value to the worker, studying radiation damage in quartz. With regard to the substitutional aluminum content, it must be noted that the concentration in a number of specimens grown under nominally similar conditions will differ slightly and partly as a result of the statistical nature of the process and also as a result of variations in the purity of the nutrient.

b. Controlled amounts of the impurities present in natural quartz can, in certain instances, be introduced. This can be of considerable assist in identifying the nature of those color centers which are impurity-dependent.

c. By the introduction of impurities not found in natural quartz, material with new properties can be grown. For example, if sufficient chromium can be introduced into the green quartz it may be possible to obtain quartz which is paramagnetic. 


\section{References}

1. T Suzuki, H Hojo, T Kobayashi (2015) Temperature calibration of quartz oscillator for outdoor hydrogen sensing. Vacuum 121: 255- 259.

2. A Suzuki (2016) Humidity calibration for a pressure gauge using a temperature-stable quartz oscillator. Applied Science and Convergence Technology 25(6): 124- 127.

3. P Armand, A Lignie, M Beaurain, P Papet (2014) Flux-grown piezoelectric materials: application to $\alpha$-quartz analogues. Crystals 4(4): 168- 189.

4. O Cambon, J Haines (2017) Hydrothermal crystal growth of piezoelectric $\alpha$-quartz phase of AO2 $(\mathrm{A}=\mathrm{Ge}, \mathrm{Si})$ and $\mathrm{MXO} 4(\mathrm{M}=\mathrm{Al}, \mathrm{Ga}, \mathrm{Fe}$ and $\mathrm{X}=\mathrm{P}$, As): a historical overview. Crystals 7(2): 38.

5. ML Chithambo, P Niyonzima (2017) Radioluminescence of annealed synthetic quartz. Radiation Measurements 106: 35-39.

6. H Kobayashi, K Horikawa, K Tanigaki, K Ogawa (2016) Impact compressive fracture of synthetic quartz accompanied by electromagnetic phenomenon. Key Engineering Materials 715: 13- 20.

7. HE Bömmel, WP Mason, AW Warner (1956) Dislocations, relaxations, and an elasticity of crystal quartz. Physical Review 102(1): 64-71.
8. E Lewicka, W Franus (2014) Study of the reasons for heterogeneity in feldspar-quartz material after firing. Mineral Resources Management 30(1): 69- 83.

9. RS Dickson, JA Weil, PH Davis (1991) The paramagnetic germaniumsodium impurity centres and in $\alpha$-quartz. Canadian Journal of Physics 69(7): 761- 779.

10. D Grasse, O Kocar, J Peisl, SC Moss (1982) Diffuse X-ray scattering from neutron irradiated crystalline quartz. Radiation Effects 66(1-2): 61- 71.

11. K Jamshidi-Ghaleh, ES Lotfi, H Masalehdan (2011) Darkening enhancement in SK3 glass with quartz under irradiation of femtosecond laser pulses. Optics and Lasers in Engineering 49(5): 605- 608.

12. GW Ratheneau (1955) Report of the conference on defects in crystalline solids. Acta Crystallographica 8(12): 855- 856.

13. L Aljerf (2018) High-efficiency extraction of bromocresol purple dye and heavy metals as chromium from industrial effluent by adsorption onto a modified surface of zeolite: kinetics and equilibrium study. Journal of Environmental Management.

14. MI Samoylovich (1982) Optical spectrum characteristics of synthetic amethyst crystal. International Geology Review 24(1): 41- 43.

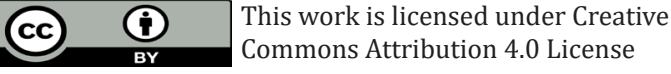

To Submit Your Article Click Here: Submit Article

DOI: 10.32474/AOICS.2018.03.000164

\section{AOICS}

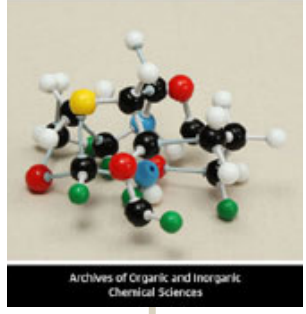

Archives of Organic and Inorganic Chemical Sciences

\section{Assets of Publishing with us}

- Global archiving of articles

- Immediate, unrestricted online access

- Rigorous Peer Review Process

- Authors Retain Copyrights

- Unique DOI for all articles 